\title{
Extended IEEE 802.11p using distance-based grouping algorithm
}

\author{
Baul Lee $^{1^{*}}$ and Jin-Yeong $\mathrm{Um}^{2}$ \\ Researcher, Department of Computer Science and Engineering, Dongguk University, South Korea ${ }^{1}$ \\ Professor, Department of Computer Science and Engineering, Dongguk University, South Korea ${ }^{2}$
}

Received: 31-May-2018; Revised: 29-July-2018; Accepted: 5-October-2018

C2019 Baul Lee and Jin-Yeong Um. Published by ACCENT Social and Welfare Society. This is an open access article distributed under the Creative Commons Attribution (CC BY) License, which permits unrestricted use, distribution, and reproduction in any medium, provided the original work is properly cited.

\begin{abstract}
Vehicle communication is being utilized to provide a variety of services in the area of intelligent transport systems (ITS) by merging the communication systems and the automotive industry. In general, vehicle communication is used for vehicle-to-vehicle (V2V) and vehicle-to-infra (V2I) communications by adopting the IEEE 802.11p / 1609 standard known as wireless access in vehicular environments (WAVE). In intelligent traffic system, vehicle information is collected by exchanging information between the roadside unit (RSU) and the vehicle. The vehicle density within the range of one $R S U$ changes with time. The higher the vehicle density, the more vehicle information is collected on a single RSU and the probability of packet collision increases. In this paper, we analyze the vehicle to infrastructure (V2I) and vehicle to vehicle (V2V) wireless channel characteristics of the WAVE communication system based on IEEE 802.11p standard in domestic urban and highway environments to solve the phenomenon that the communication performance is deterioration when concentrated on this one infrastructure. When there are multiple vehicles communicating with a single infrastructure, the collision rate increases, which makes communication difficult. In this paper, we propose a distance-based grouping algorithm that groups multiple vehicles and communicates between groups. By using the algorithm proposed in this paper, the collision rate is reduced by an average of $4.2 \%$.
\end{abstract}

\section{Keywords}

IEEE 802.11p, IEEE 1609.4 wireless access vehicular environments, Vehicle network, Collision probability, Vehicle clustering, Grouping.

\section{Introduction}

Advances in technology have made cars an indispensable factor in our lives. Wireless payment systems are already in use, providing convenient facilities for vehicles, various media services available in vehicles and wireless toll payment systems. As demands for multimedia services and vehicle safety have increased in recent years, more and more attempts have been made to use the services while moving. Research continues on intelligent transportation system (ITS) for smart vehicles with access to safety and entertainment services in the United States, Europe and Japan leading the way [1]. The field of vehicle communication in ITS technology is recognized as an important part of providing a variety of services to ensure the reliability and safety of the vehicle and the road environment. IEEE 802.11p and 1609, the nextgeneration vehicle protocols, are the wireless access in vehicular environments (WAVE) communication.

*Author for correspondence

61
WAVE consists of physical (PHY) layer and Media Access Control (MAC) layer of IEEE 802.11a and is composed of additional functions such as resource allocation, security and multi-channel service supported in 1609. Supports quality of service (QoS) of each data stream using multiple channels of IEEE 1609.4 and enhanced distributed coordination function channel access (EDCA) of IEEE 802.11e [2].

IEEE 802.11p uses the distributed coordination function (DCF) method used in the MAC by IEEE 802.11 and is based on carrier-sense multiple access with collision avoidance (CSMA / CA). CSMA / CA is a method of avoiding a collision in wireless communication. Also, QoS is supported by assigning different priorities to packets generated according to an application by using EDCA extended from DCF of IEEE 802.11. IEEE 802.11p / WAVE used consists of a vehicle and a road side unit (RSU) installed on the road [3]. By vehicle, we mean one 
that is equipped with an on-board-unit (OBU) supporting wireless communication in the vehicle and participating in the vehicular ad-hoc network (VANET). An RSU is a device that supports communication around the road and is also referred to as an infrastructure. VANET communication can be roughly classified into a vehicle to infrastructure (V2I), which is a communication between a vehicle and an infrastructure, and vehicle to vehicle (V2V), which is a communication between a vehicle and a vehicle.

As the number of vehicles increases when a vehicle communicates with one RSU, there is a problem of intensifying competition and collision. In order to avoid competition and collision, existing researches have added data to transmit in order to stabilize communication, but data transmission is difficult for vehicles with high mobility due to large communication overhead. In this paper, we use the hidden-node-aware grouping (HAG) algorithm, which is a grouping algorithm in existing mobile wireless network environment, to the vehicle environment. In the conventional vehicle communication, the distance between the vehicles is obtained by using the position information of each vehicle, the vehicles having a short distance between the vehicles are preferentially grouped, and the time is allocated to each group to reduce the collision rate.
Vehicle grouping elects a group leader and one or more group members are present in the group to allow intragroup communication and inter-group communication.

This paper is composed as follows. Section 2 describes the WAVE communication, IEEE 802.11p and IEEE 1609.4. Section 3 describes the HAG algorithm and Section 4 explains the distance-based grouping algorithm that is modified by the HAG algorithm. Section 5 presents the performance evaluation of the proposed algorithm and conclusion.

\section{WAVE communication}

WAVE is a vehicle to everything (V2X) vehicle networking technology for public safety and ITS services. The existing communication protocol used in ITS service is dedicated-short-rangecommunication (DSRC) as a short-distance communication method. It is used mainly for highpass fare collection service, bus route identification and traffic information notification service for navigation. In order to solve this problem, IEEE has developed WAVE communication, including IEEE 802.11p and IEEE1609 because there is a limit to accommodate various services and high transmission speed required in ITS including wireless internet. The standard structure of WAVE is shown in Figure 1 [4].
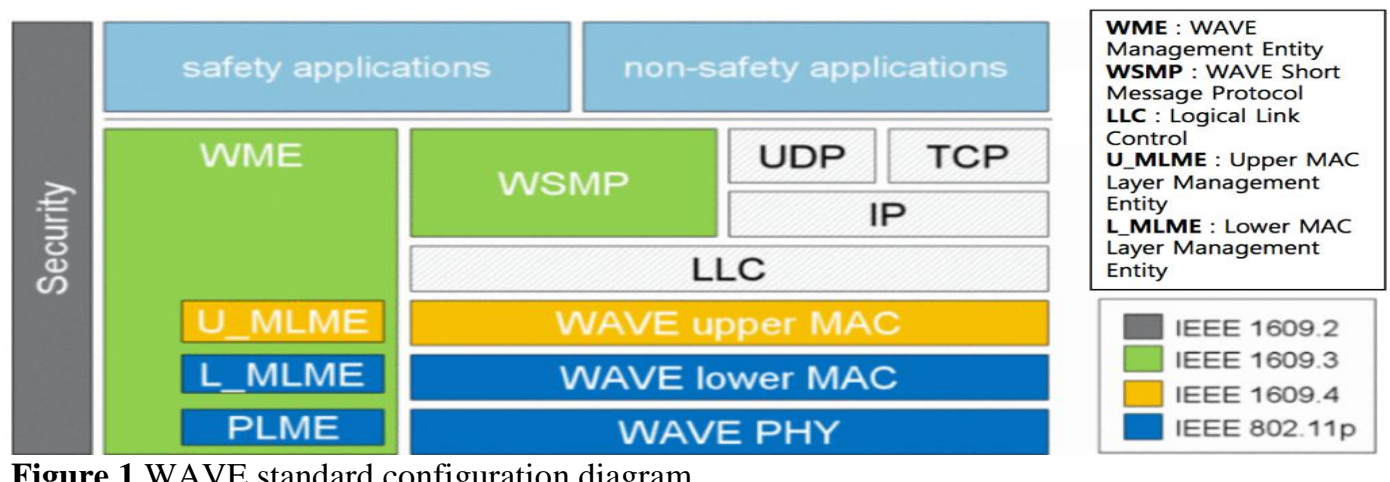

Figure 1 WAVE standard configuration diagram

\subsection{IEEE 802.11p}

The IEEE 802.11p standard is meant to:

- Describe the functions and services required by WAVE-conformant stations to operate in a rapidly varying environment and exchange messages without having to join a basic service set (BSS), as in the traditional IEEE 802.11 use case.

- Define the WAVE signaling technique and interface functions that are controlled by the IEEE 802.11 MAC.
IEEE 802.11p defines WAVE communication considering the existing IEEE 802.11a based mobile environments [5]. Vehicle communication has short communication time than general wireless network because of high-speed mobility which is characteristic of vehicle and road environment based on vehicle communication. Due to the short communication connection time, IEEE 802.11p omits the combining and authentication procedures defined in the existing IEEE 802.11a. In the BSS mode in which the communication node communicates with 
the access point (AP), the node using IEEE 802.11a hears the beacon message transmitted by the AP and performs authentication and association procedures. After completing the authentication and association procedure, the AP and the node form a BSS. However, IEEE 802.11p, which has a relatively short communication time, uses the WAVE mode in which authentication and association procedures are omitted and a BSS is formed [6]. Any two wireless terminals operating in the WAVE mode can exchange data if the channels coincide. The BSS formed in the WAVE mode is called WAVE basic service set (WBSS).

A beacon is transmitted from a node having transmission data of IEEE 802.11p WAVE

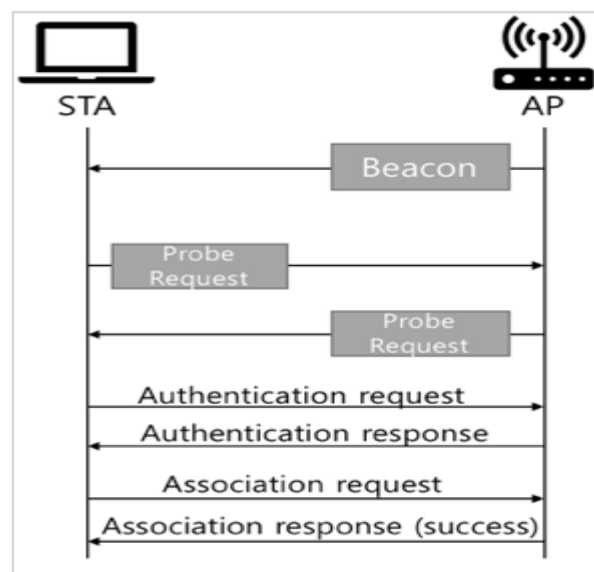

(a) communication, and a WBSS is formed together with the node receiving the beacon. The nodes subscribed to the WBSS can send and receive data without authentication and association procedures. At this time, the beacon frame provides necessary information at the higher layer of IEEE 802.11p and provides information for the service and subscription provided by WBSS. Figure 2 shows the BSS formation process of IEEE 802.11a and the WBSS formation process of IEEE 802.11p [7].

Figure 2 (a) IEEE 802.11a BSS subscription process (b) IEEE 802.11p WBSS subscription process

IEEE 802.11p WBSS eliminates authentication and association procedures, so the problem that arises is addressed in the IEEE 1609 standard.

\subsection{IEEE 1609.4}

IEEE 1609.4 supports multi-channel operations. The multi-channel architecture of WAVE MAC basically guarantees Quality of Service (QoS) for each AC (Access Category) by using EDCA (Enhanced Distributed Channel Access) mechanism of IEEE 802.11e [8].

The CCH channel is a channel for transmitting safety messages, and there are periodic sending status messages and event-based emergency messages that occur in an emergency situation [9]. The $\mathrm{SCH}$ channel is a channel for exchanging safety messages and general data. Figure 3 shows the multi-channel architecture of WAVE MAC [4].

\section{HAG algorithm}

HAG algorithm is an algorithm for eliminating interference collision in mobile wireless network environments [10]. This section introduces the HAG algorithm to be used in the proposed algorithm. The HAG algorithm is divided into five steps. The step of recognizing the hidden node state at the first step, the step of grasping the hidden node at step 2, the step of generating the signal strength table at step 3, the step of applying the grouping algorithm at step 4 and the step of allocating the channel at each step shown in Figure 4. 


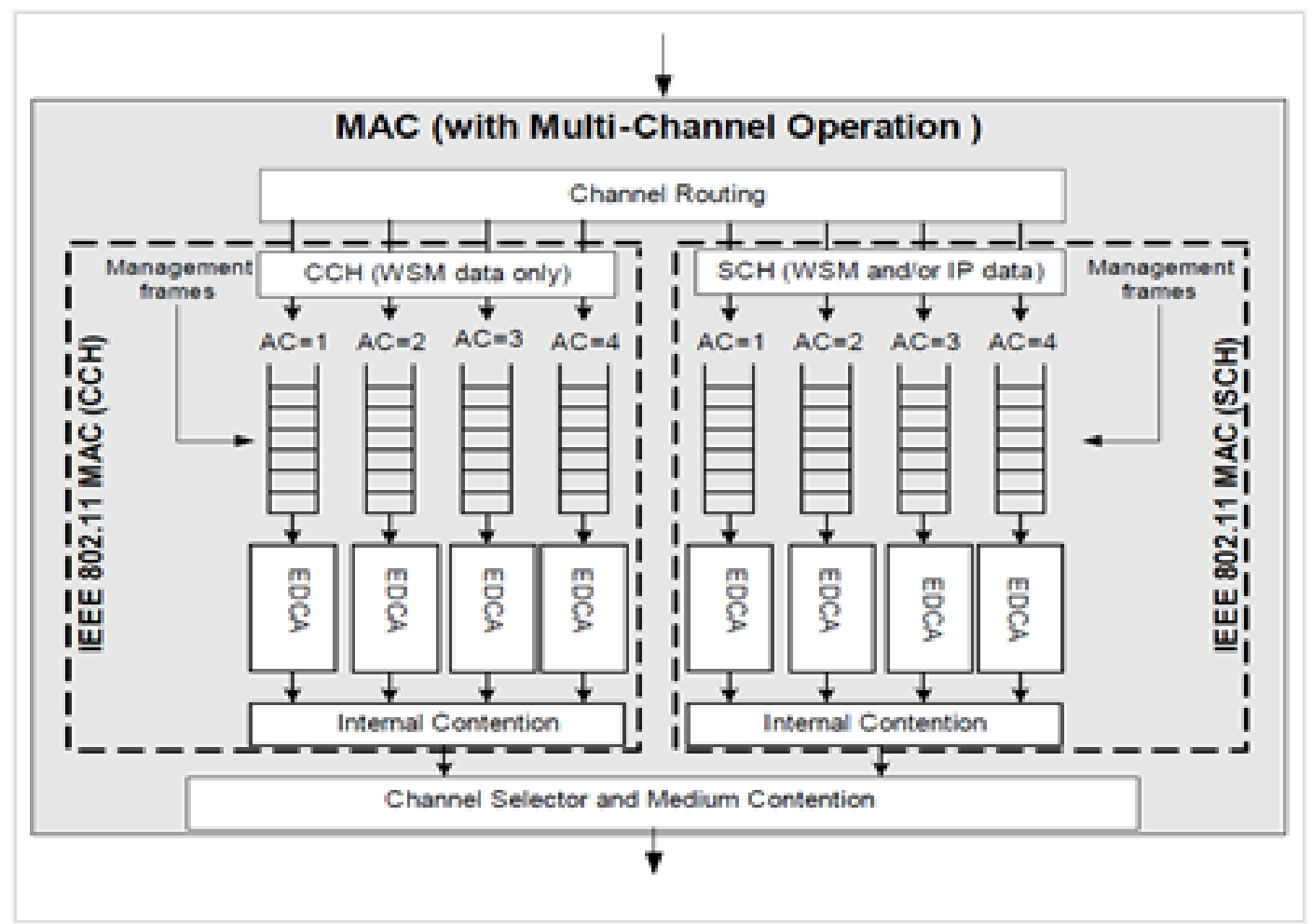

Figure 3 WAVE MAC multi-channel architecture

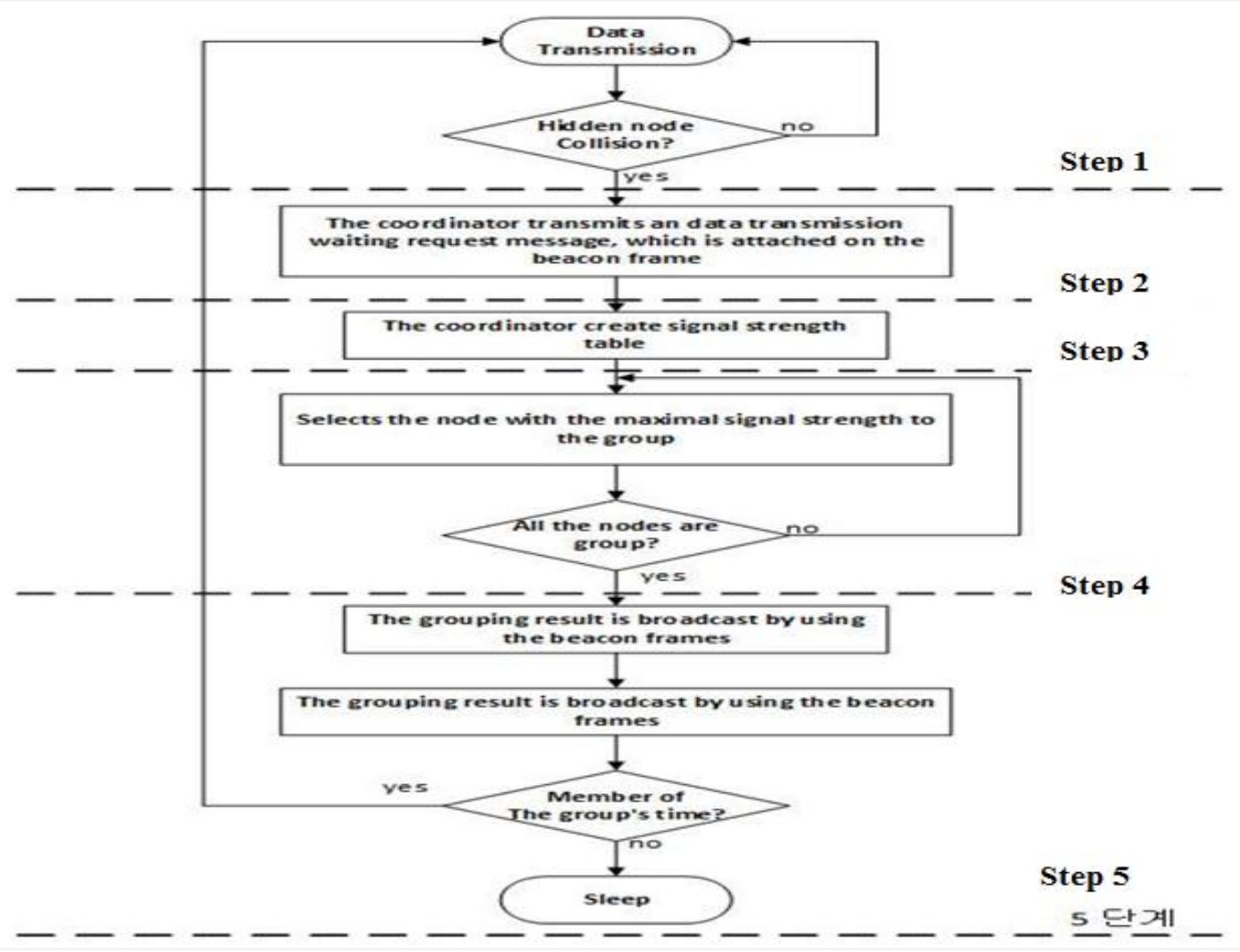

Figure 4 HAG algorithm flowcharts 
Figure 5 shows the HAG algorithm's identification step and signal strength acquisition process

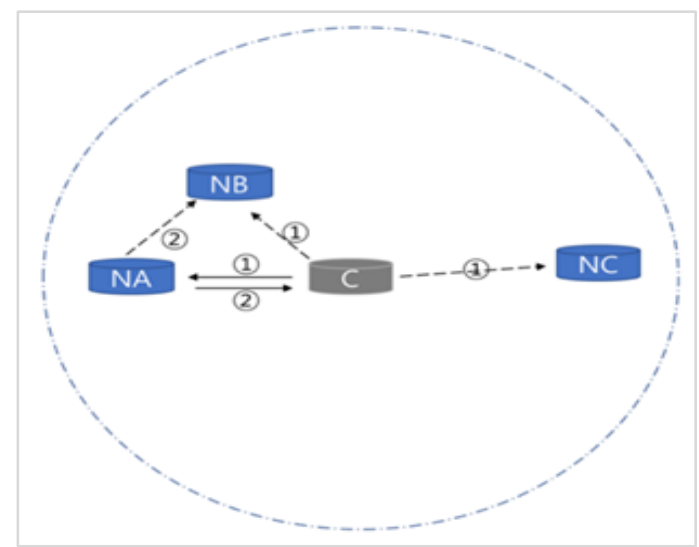

Figure 5 HAG algorithm's identification step and signal strength acquisition process

In the first step of the HAG algorithm, a case where the coordinator detects a collision while receiving a signal from one node is defined as a collision due to the hidden node. The coordinator that detects the collision due to the hidden node becomes aware of the existence of the hidden node. The coordinator recognizing the hidden node enters the second-step hidden node relation grasp step. Assume that there are coordinator $\mathrm{C}$ and nodes $\mathrm{NA}, \mathrm{NB}$, and $\mathrm{NC}, \mathrm{NA}$ and $\mathrm{NB}$ are in the same transmission range, and $\mathrm{NC}$ is in a position outside the transmission range of NA and NB. When $\mathrm{C}$ sends a message to NA, NA and NB can both hear the message. In this case, if the NA transmits an ACK to $\mathrm{C}$ in response to the message, NB records the ACK and records the signal. Since $\mathrm{NC}$ cannot hear, the signal strength is recorded as -1 . In the third step, the signal strength table generation step, the coordinator transmits a frame requesting to report the signal strength recorded by each node, receives a response, and generates a signal strength table. Apply the four-step grouping algorithm using the prepared signal intensity table. Start with the two nodes with the strongest signal in the signal table and make the node and group with the highest signal strength. After the group is created, the process goes to step 5, which is a group-by-group channel allocation step. As the size of each group increases, the allocated transmission time increases.

\section{Distance-based grouping algorithm}

The WAVE communication used in the VANET increases the data collision rate when a large number of vehicles are concentrated in one RSU. We propose a distance-based grouping algorithm [11] to reduce the collision rate even in the presence of multiple vehicles. In the distance-based grouping algorithm proposed in the background of the HAG algorithm described above, a table is created by replacing the signal strength parameter value used by the HAG algorithm with the inter-vehicle distance value. If the criterion for applying the HAG algorithm to the VANET is the signal strength of the node, it is not suitable for vehicle communication requiring fast communication because each vehicle needs a procedure for receiving and confirming the signal. Therefore, this paper proposes a grouping algorithm based on the distance between vehicles.

The proposed algorithm can be divided into three stages. In the first step, the distance information between the vehicles is stored in the RSU that knows all the vehicle nodes. In the second step, the group is created based on the distance using the created table and the group leader is selected. The last three steps consist of transferring data from a given group. This chapter consists of four sections. Section 1 describes the overall algorithm. Section 2 describes the RSU table creation process, and Section 3 describes the grouping process using tables. In the last section, we describe the communication process of the grouped nodes.

\subsection{Grouping algorithm}

The algorithm can be divided into three stages. The overall grouping and group communication process is shown in Figure 6.

In step 1, the RSU updates the information on all the vehicles in the transmission range to the RSU table, checks the collision rate, and checks whether grouping is required. If the collision rate exceeds the threshold value, it is judged that the grouping should proceed and then step 2 is entered. Step 2 is a grouping and group communication process. The RSU creates a grouping table by calculating the distance between each vehicle based on the RSU table that has been created previously. Using the grouping table in which the distances between vehicles are written, the nearest vehicles are grouped together, and the group leader is selected as the slower vehicle. After repeating the process of creating a group, when a vehicle belonging to an arbitrary group exceeds 20 , which is a criterion for decreasing the throughput value, the entire grouping is stopped and the process proceeds to the group communication step 3. Experiments to measure the throughput according to the number of vehicles are conducted in Section 3. In step 3, the RSU 
communicates with the group by assigning time to each group. The vehicle may move out of the range of the RSU due to the movement of the vehicle. If the vehicle leaves the RSU, the RSU erases the vehicle from the table. If the group leader with the slow movement is out of range, the RSU re-checks the collision rate after resolving the traffic congestion on the road or recognizing it as a new vehicle inflow, clearing all groups.

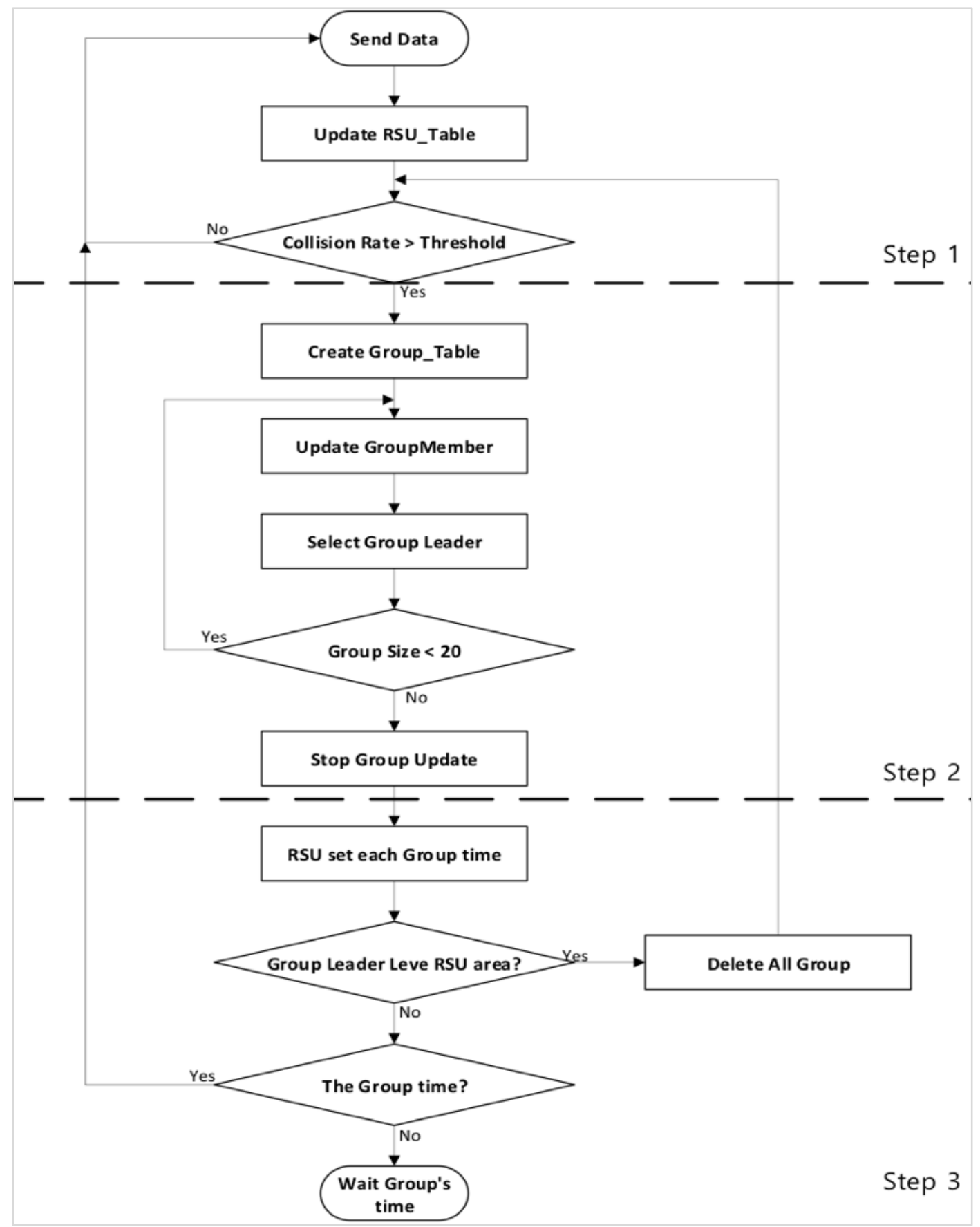

Figure 6 Distance-based grouping algorithm flowcharts 


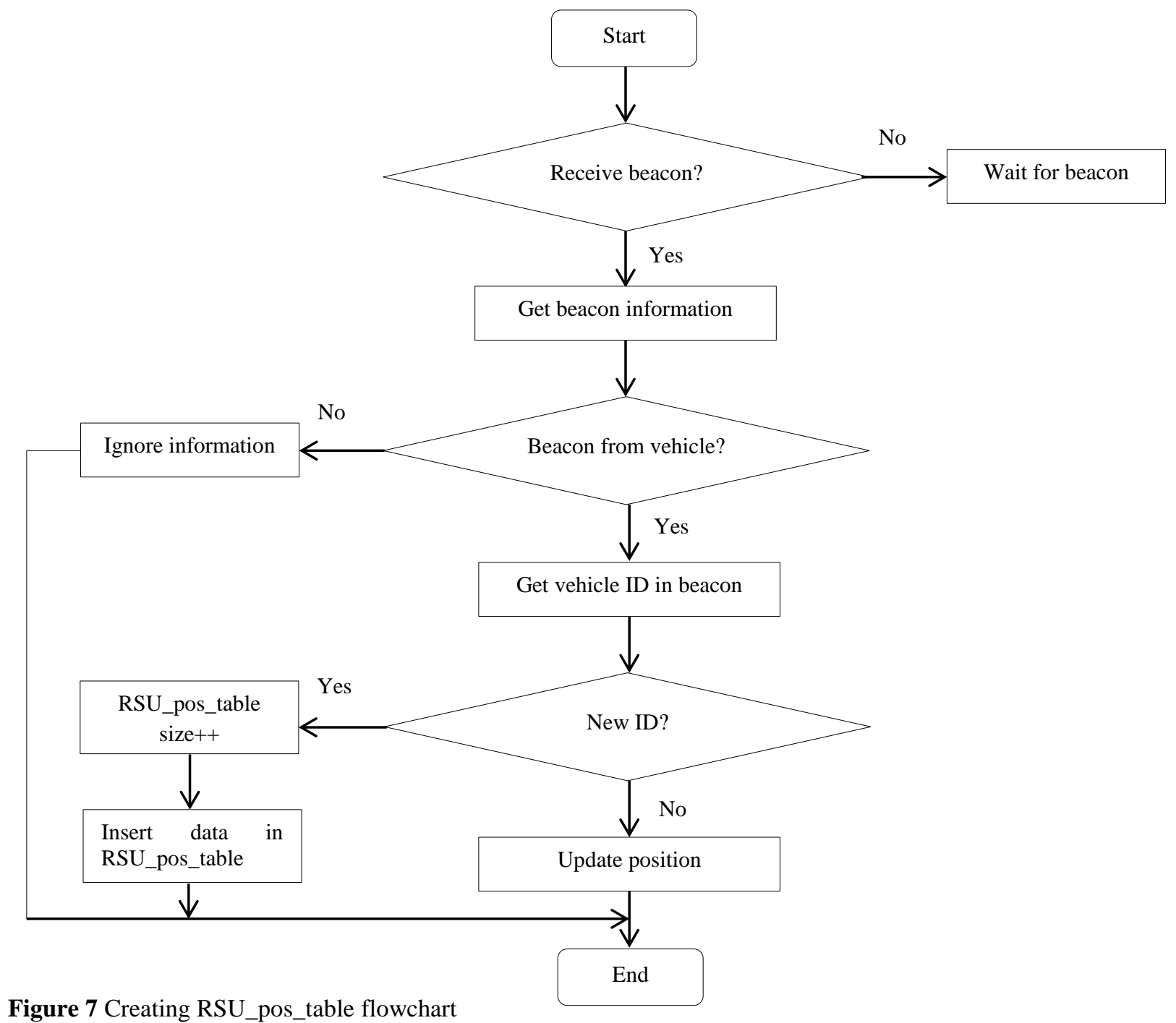

\subsection{Create the table for grouping}

The RSU and the vehicle exchange information through a beacon message. The RSU stores the information contained in the received vehicle beacon message for later grouping steps. The RSU has two tables, the first is a table (RSU_pos_table) with vehicle location information, and the second is a table (RSU_dist_table) that calculates and stores the distance between vehicles. Figure 7 shows the process of creating RSU_pos_table. Each time the RSU receives a beacon message from the vehicle, it stores the location information of the vehicle in the RSU_pos_table. If the vehicle ID contained in the Beacon message is an already created ID, the location is updated.
After creating the RSU_pos_table, create an RSU_dist_table that represents the distance using the location information in the RSU_pos_table. A twodimensional array type RSU_dist_table having a size equal to the number of IDs in the RSU_pos_table is created, and the distance between the vehicles is obtained and stored in the RSU_dist_table. For example, when vehicles of the $\mathrm{V} 0$ and $\mathrm{V} 1$ are present, the IDs and positions of the $\mathrm{V} 0$ and $\mathrm{V} 1$ are stored in the RSU_pos_table. The distance dist (V0, $\mathrm{V} 1)$ between $\mathrm{V} 0$ and $\mathrm{V} 1$ is obtained using the location information stored in the RSU_pos_table and stored in the RSU_dist_table. Figure 8 shows an example of the created RSU_dist_table. 

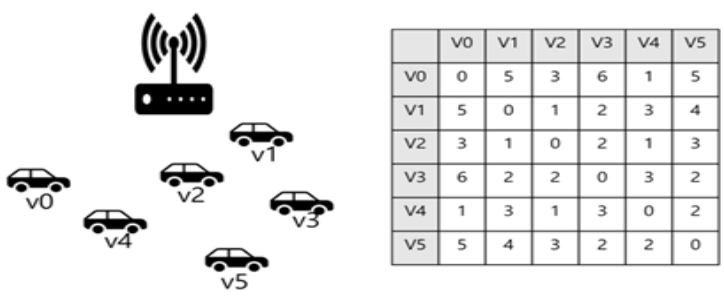

Figure 8 Created RSU_dist_table by RSU

\subsection{Group and group leader selection}

Before dividing the group based on the RSU_dist_table, it is necessary to confirm whether grouping is necessary. The RSU calculates the collision rate after creating the table. If the collision rate does not exceed the threshold value, it is determined that grouping is not necessary, and then the RSU table is updated again after the data transmission.
If the collision rate exceeds the threshold value, the RSU determines that grouping is necessary and carries out distance-based grouping between the vehicles. The composition of a group is divided into a group leader and a group member. As the leader of the group moves slower, the leader of the group stays within the range of the RSU longer. Therefore, the group leader is selected as the slower vehicle that can stay stable in the RSU for a long time.

Groups are created based on the RSU_dist_table created by the RSU, and the vehicles located close to each other are grouped first. Create a group by grouping the vehicles to the nearest two nodes and create the table again. At this time, among the two vehicles that are grouped, a relatively slow vehicle is selected as a group leader, and the vehicle is set as a standard for distance to other vehicles and groups.

\begin{tabular}{|l|l|l|l|l|l|l|}
\hline & 0 & 1 & 2 & 3 & 4 & 5 \\
\hline 0 & 0 & 5 & 3 & 6 & 1 & 5 \\
\hline 1 & 5 & 0 & 1 & 2 & 3 & 4 \\
\hline 2 & 3 & 1 & 0 & 2 & 1 & 3 \\
\hline 3 & 6 & 2 & 2 & 0 & 3 & 2 \\
\hline 4 & 1 & 3 & 1 & 3 & 0 & 2 \\
\hline 5 & 5 & 4 & 3 & 2 & 2 & 0 \\
\hline
\end{tabular}

(a)

\begin{tabular}{|c|c|c|c|}
\hline & 0,4 & 1,2 & 3,5 \\
\hline 0,4 & 0 & 3 & 2 \\
\hline 1,2 & 3 & 0 & 4 \\
\hline 3,5 & 2 & 4 & 0 \\
\hline
\end{tabular}

(b)

\begin{tabular}{|c|c|c|}
\hline & 0,4 & 1,2 \\
\hline 0,4 & 0 & 4 \\
\hline 3,5 & 0 & 0 \\
\hline 1,2 & 4 & 0 \\
\hline
\end{tabular}

(c)

Figure 9 Group decision process using RSU_dist_table

For example, Figure 9 shows the RSU_dist_table created by the RSU. There is a total of six vehicles on the road, and each vehicle is at a distance as shown in Figure $9(a) .(1,2)$ and $(3,5)$ are grouped as the group with the closest distance between vehicles $(0$, 4 ), and the table is updated as shown in Figure $9(b)$. Assuming that 1,4 , and 5 in each group are the vehicles with the lowest speed in the group, 1,4 , and 5 become the group leader and serve as a basis for comparing distances from other groups.
We examine the distance between leader and the leader of each group and perform the merging between groups as shown in Figure 9 (c). A group was simulated by increasing the number of nodes in the IEEE $802.11 \mathrm{p}$ standard to have the maximum throughput. As shown in Figure 10, when the number of vehicles was increased and simulated, the number of vehicles showed the highest throughput at 20 vehicles. Therefore, the maximum allowable number of nodes per group is 20 .

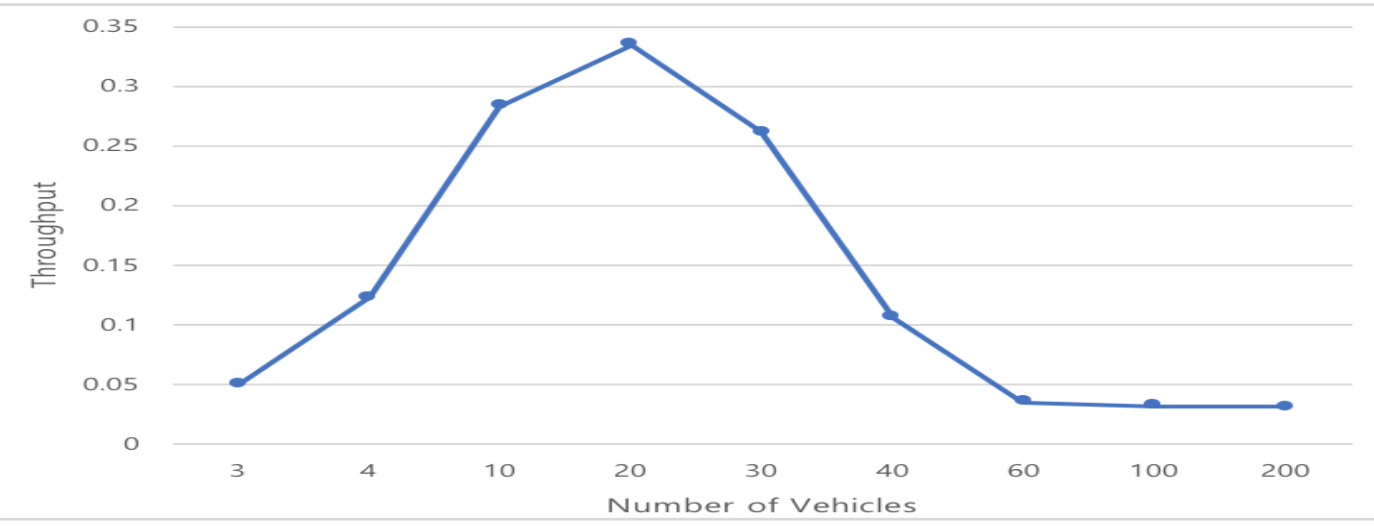

Figure 10 Throughput graph according to the number of vehicles 


\section{Performance evaluation}

We used OMNET ++ to evaluate the performance of IEEE 802.11p. We modified the communication procedure using vehicular network open simulator (VENTOS) to create the scenario of the vehicle environment. We have connected the simulation of urban mobility (SUMO). Through sumo simulation, the vehicle is given mobility and the vehicle is recognized to OMNET++ and bound to communicate. The simulation environment was Linux Ubuntu 16.04 LTS, OMNET ++ 5.1, and SUMO 2.90. The network simulation parameters are shown in Table 1.

Table 1 Simulation parameter

\begin{tabular}{ll}
\hline Parameters & Value \\
\hline Data rate & $6 \mathrm{Mbps}$ \\
Beacon size & $390 \mathrm{bits}$ \\
Data packet size & $8326 \mathrm{bits}$ \\
RSU beacon interval & $1 \mathrm{~s}$ \\
Vehicle beacon interval & $0.1 \mathrm{~s}$ \\
CW min & 16 \\
CW max & 1024 \\
Slot time & $9 \mathrm{us}$ \\
Number of vehicle & $3,10,20,30$, \\
& $40,60,100,200$ \\
Road lane & 4 \\
\hline
\end{tabular}

In a vehicle simulation environment, the vehicle and the RSU always have data to transmit. The inflow and the flow of the vehicle are Poisson distribution and set a variable speed instead of a constant speed and set it similar to the actual road situation. It is assumed that the assumed road situation in the simulation situation is a unidirectional four lane road and that all the roads included in the simulation are within the range of RSU. The vehicle is always in a state of having data to send, and it sends and receives beacon messages to and from the RSU and other vehicles. Vehicle communication is not smooth because of the high collision rate in urban environments or on stagnant highways where many vehicles are driven.

Figure 11 is a graph comparing the proposed IEEE 802.11 p / 1609 with the collision rate by applying the proposed distance-based grouping to vehicle communication. The proposed algorithm and the existing communication have an average of $4.2 \%$ lower collision rate of the proposed algorithm based on 20 nodes where grouping starts.

Figure 12 compares the throughput of the proposed algorithm with the existing communication using IEEE 802.11p / 1609. The existing communication and the proposed distance - based grouping algorithm show the difference in performance from 20 cars or more.

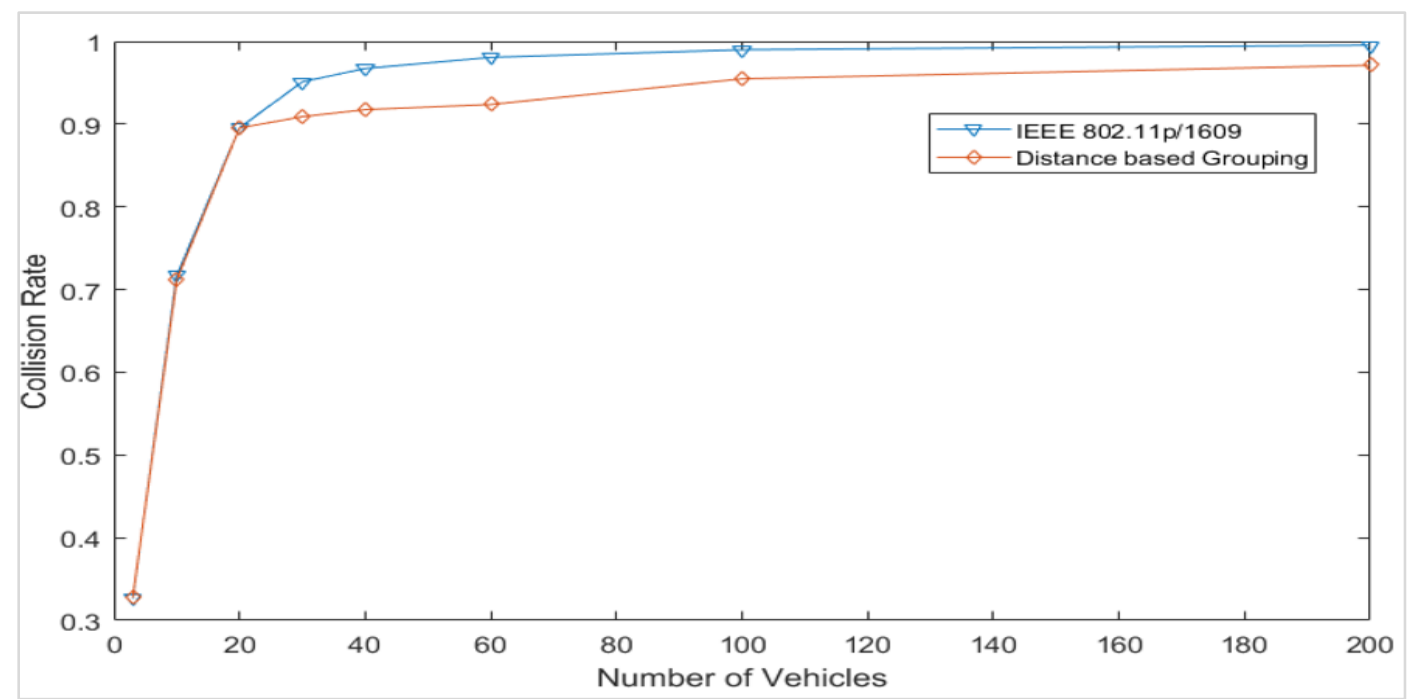

Figure 11 Comparison of collision rate between IEEE 802.11p/1609 and using distance-based grouping algorithm 
Baul Lee and Jin-Yeong Um

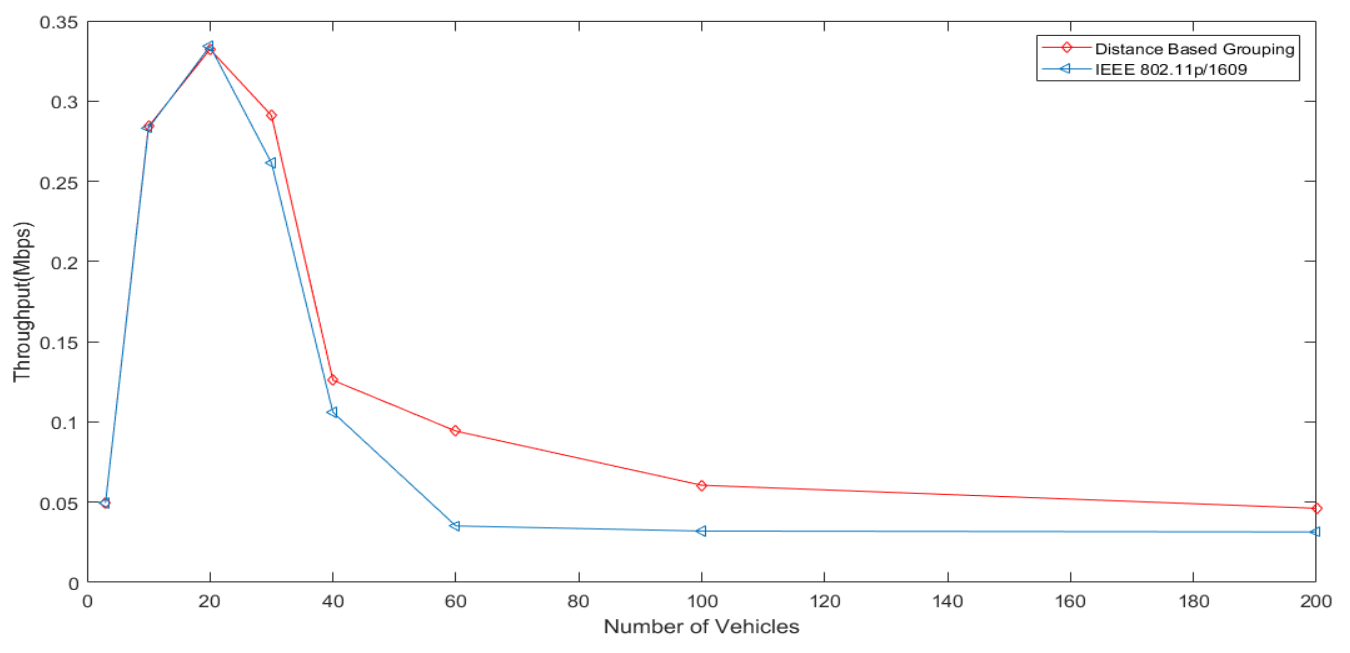

Figure 12 Throughput of distance-based grouping algorithm and IEEE 802.11p / 1609

\section{Conclusion}

IEEE $802.11 \mathrm{p}$ standard communications show that as the number of vehicles increases, the collision rate increases, so the throughput decreases significantly after the number of vehicles exceeds 20 vehicles. Using the proposed algorithm, vehicle performance is improved by a maximum of $5.9 \%$, a minimum of $1.47 \%$, and an average of $3 \%$.

In this paper, thresholds were limited to 20 per group as an experimental result. In the future, the RSU will attempt to create an algorithm that will determine the group resources according to the number of vehicles on the road and optimize the collision rate and the transmission rate.

\section{Acknowledgment}

This research was supported by the Basic Science Research Program through the National Research Foundation of Korea (NRF) funded by the Ministry of Education (No. 2017R1D1A1B03032736).

\section{Conflicts of interest}

The authors have no conflicts of interest to declare.

\section{References}

[1] Alam M, Ferreira J, Fonseca J. Introduction to intelligent transportation systems. In intelligent transportation systems 2016 (pp. 1-17). Springer, Cham.

[2] IEEE 1609 working group. IEEE guide for wireless access in vehicular environments (WAVE)architecture. IEEE Std. 2014: 1609-0.
[3] Qiu HJ, Ho IW, Chi KT, Xie Y. A methodology for studying $802.11 \mathrm{p}$ VANET broadcasting performance with practical vehicle distribution. IEEE Transactions on Vehicular Technology. 2015; 64(10):4756-69.

[4] IEEE 1609 working group. IEEE standard for wireless access in vehicular environments (WAVE)-multichannel operation. IEEE Std. 2016:1609-4.

[5] Eichler S. Performance evaluation of the IEEE 802.11 $\mathrm{p}$ WAVE communication standard. In vehicular technology conference 2007 (pp. 2199-203). IEEE.

[6] Jiang D, Delgrossi L. IEEE 802.11 p: towards an international standard for wireless access in vehicular environments. In vehicular technology conference 2008 (pp. 2036-40). VTC spring. IEEE.

[7] Lottermann C, Botsov M, Fertl P, Müllner R, Araniti G, Campolo C, et al. Vehicular ad hoc Networks: Standards, Solutions, and Research. 2015.

[8] Shen Y, Jeong J, Oh T, Son SH. CASD: a framework of context-awareness safety driving in vehicular networks. In international conference on advanced information networking and applications workshops 2016 (pp. 252-7). IEEE.

[9] Abbad SA, Godse SP. Priority based emergency message forwarding scheme for time critical models in VANET. In international conference on advances in electronics, communication and computer technology 2016 (pp. 393-8). IEEE.

[10] Um JY, Ahn JS, Lee KW. Evaluation of the effects of a grouping algorithm on IEEE 802.15. 4 networks with hidden nodes. Journal of Communications and Networks. 2014; 16(1):81-91.

[11] Bilgin BE, Gungor VC. Performance comparison of IEEE $802.11 \mathrm{p}$ and IEEE $802.11 \mathrm{~b}$ for vehicle-tovehicle communications in highway, rural, and urban areas. International Journal of Vehicular Technology. 2013. 


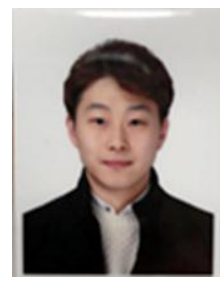

Baul Lee is in the Department of Computer Engineering, Dongguk University, Seoul, South Korea. He received the M.S. degrees in Computer Engineering from Dongguk University in 2018. His major interests are Smart and Connected Vehicle Network, Wireless Networks, and Network Simulation Techniques.

Email: ba93love@gmail.com

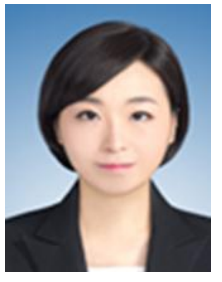

Jin-Yeong Um is in the Department of Computer Engineering, Dongguk University, Seoul, South Korea. He received the Ph.D., M.S., and B.S. degrees in Computer Engineering from Dongguk University in 2013, 2008, and 2004, respectively. Her major interests are Smart and Connected Vehicle Network, Wireless Sensor Networks, and Network Simulation Techniques.

Email:mog07@dongguk.edu 